\title{
La relación entre la responsabilidad social y la rendición de cuentas, desde la percepción del empresario de la provincia de Imbabura-Ecuadors
}

\author{
The relationship between social responsibility and accountability from the \\ perception of entrepreneurs in the Imbabura province of Ecuador
}

\section{Doi: 10.22458/rna.v1 1i2.2726}

\author{
Mag. Wilmer Medardo Arias Collaguazo \\ Universidad Regional Autónoma de Los Andes, Ecuador \\ ui.wilmerarias@uniandes.edu.ec \\ https://orcid.org/0000-0002-1438-4012
}

\section{RESUMEN}

La responsabilidad social se asume como un compromiso voluntario de las empresas, para asegurar un crecimiento económico, social y ambientalmente responsable por lo cual es necesario, determinar la percepción de los empresarios con respecto a la rendición de cuentas por sus actividades económicas realizadas, estudio que se enmarca dentro de la modalidad cualitativa y cuantitativa, aplicando métodos de nivel teórico, para el diagnóstico a varias empresas en la provincia de Imbabura-Ecuador, aplicándose la técnica de la encuesta, como resultados importantes de la percepción de los empresarios es que no hay suficiente compromiso en lo respecto a la rendición de cuentas.

\begin{abstract}
Social responsibility is assumed as a voluntary commitment of companies to ensure economic, social and environmentally responsible growth for which it is necessary to determine the perception of entrepreneurs regarding accountability for their economic activities. This study is framed within the qualitative and quantitative modality, applying theoretical level methods, for the diagnosis to several companies in the province of Imbabura-Ecuador. The survey technique is used in relation to the perception of entrepreneurs. As a result, it is noted that there is not enough commitment regarding accountability.
\end{abstract}

\section{RÉSUMÉ}

La responsabilité sociale est assumée comme un engagement volontaire des entreprises pour assurer une croissance économique, sociale et environnementale responsable, afin de déterminer la perception des entrepreneurs en matière de reddition de comptes sur leurs activités économiques. Cette étude s'inscrit dans un cadre qualitatif et quantitatif, en appliquant des méthodes de niveau théorique pour le diagnostic de plusieurs entreprises de la province d'Imbabura en Équateur. La technique de sondage est utilisée au sujet de la perception des entrepreneurs. Il en ressort qu'il n'y a pas assez d'engagement en matière de reddition de comptes.

\section{RESUMO}

A responsabilidade social é assumida como um compromisso voluntário das empresas, para garantir o crescimento econômico, social e ambientalmente responsável pelo qual é necessário, para determinar a percepção dos empresários em relação à responsabilização por suas atividades econômicas, um estudo que Está enquadrado na modalidade qualitativa e quantitativa, aplicando métodos de nível teórico, para o diagnóstico de várias empresas da província de Imbabura-Equador, aplicando a técnica de pesquisa, pois resultados importantes da percepção dos empreendedores é que não há Compromisso suficiente em relação à prestação de contas.

\section{PALABRAS CLAVE:}

POLIITICA Y BIENESTAR SOCIAL, RESPONSABILIDAD SOCIAL, COMPORTAMIENTO SOCIAL, NECESIDAD SOCIAL.
KEY WORDS:

SOCIAL POLICY AND

WELFARE, SOCIAL

RESPONSIBILITY, SOCIAL

BEHAVIOR, SOCIAL ED.
PALAVRAS CHAVE:

POLITICA E BEM-ESTAR

SOCIAL, RESPONSABIL-

IDADE SOCIAL, COM-

PORTAMENTO SOCIAL, NECESSIDADE SOCIAL.
MOTS CLÉS:

POLITIQUE ET BIEN-ÊTRE SOCIAL, RESPONSABILITÉ SOCIALE, COMPORTEMENT SOCIAL, BESOIN SOCIAL. 


\section{INTRODUCCIÓN}

Para tratar el tema de rendición de cuentas, es necesario profundizar en la materia de responsabilidad social empresarial y en sus orígenes que según Rauflet, Lozano, Barrera y García (2012) el concepto tuvo su origen en 1953 cuando Howard Bowen plantea por primera vez como una pregunta a la sociedad empresarial en su trabajo ¿cuáles son las responsabilidades de los empresarios?, iniciando un debate teórico de la responsabilidad de un empresario, sin embargo las acciones se materializarían décadas después, con la implementación de una normativa en firme conocida como la "La Directiva 2014/95 del Parlamento Europeo y del Consejo" (Bardel, 2019, pp. 46-48). Y aunque este concepto tuvo un largo recorrido para ser aceptado según Varela (2013); Correa (2007) ya para la década de 1960 la academia cubría temas como el desarrollo sustentable y sostenible de las empresas, respetando a la sociedad y al medio ambiente. En esta misma época Davis citado en (Monroy, 2013), propone que "la responsabilidad de las empresas se da en función de la cantidad de poder que éstas tienen sobre la sociedad" (p. 26), argumento que lleva a un intenso debate con Friedman (1970) quien afirma que la responsabilidad de los directivos no puede ser otra que aumentar los beneficios de sus accionistas; y declaró a la responsabilidad social empresarial (RSE) es una doctrina no apta para una empresa, argumentos que llevaría a desarrollar informes en la Unión Europea con enunciados donde "el principio rector de las empresas es que sean competitivas y rentables sin dejar de cumplir sus responsabilidades sociales" (Bardel, 2019, p. 36), y donde su rendición de cuentas no era todavía analizada sobre ninguna mesa de discusión.

Para el desarrollo de un informe no financiero "rendición de cuentas", se necesitó el desarrollo teórico aportado durante la década de 1975 con estudios exploratorios para establecer las características y modelos como los de Sethi (1975), formado por tres etapas o el de Carroll (1979) constituido en cuatro categorías, que han sido útiles para llevar la teoría a la práctica. Sin embargo, Drucker (1984) presenta un avance teórico para implementar la responsabilidad social en las empresas que consiste en "que los negocios deben convertir la responsabilidad social en oportunidades de negocio" (Raufflet, Lozano, Barrera, \& García, 2012, p. 5), concepto que se refleja en el desarrollo del Libro Verde "señalando que la RSE debe considerarse una inversión y no un gasto, al igual que la gestión de la calidad" (Bardel, 2019, p. 39).

Pero el mayor desarrollo hacia un informe sería con aportes de Cochran, Wood y Jones (1985), plantean que la RSE debe ser implementada bajo principios, iniciando el debate de hasta dónde debe llegar la responsabilidad con el ambiente social y natural que le rodea a la empresa, llegando a repercutir en la creación de principios y normas escritas por organismos e instituciones orientados hacia esa finalidad.

Para la década de 1990, se habla de la competitividad como factor clave en la supervivencia de las organizaciones, las cuales debe atraer a los clientes con argumentos diferenciadores, para lo cual Wood (1991) sugiere relacionar la RSE con otras teorías administrativas. En 1995, Donaldson y Preston ponen un énfasis en las relaciones y reconocen un valor intrínseco de los grupos de interés externos. Y de esta forma inicia el debate de la forma de gestionar las relaciones con los grupos de interés desde un aspecto normativo y operacional, Kramer y Porter (2002); Kotler y Lee (2005) consolidaron con un marco de referencia explicando los beneficios desde un punto de vista mercadológico.

En el 2000 la RSE integra dos paradigmas el primero donde las empresas deben lograr un modelo de desarrollo sustentable, vinculado con actores de índole pública, social, y ambiental, con un segundo paradigma en la cual las empresas deben buscar la mejora de las relaciones con sus grupos de interés reduciendo el impacto ambiental. Y es en esta década que se desarrolla el Libro Verde definiendo un concepto de Responsabilidad Social Empresarial como "la integración voluntaria, por parte de las empresas, de las preocupaciones sociales y medioambientales en sus operaciones comerciales y sus relaciones con los interlocutores" (Bardel, 2019, pp. 38-39). Definición que se traducía a un estándar mínimo, pues al ser voluntaria no era necesaria su aplicación.

Sin embargo, en las 2006 iniciativas como las de la Organización de Naciones Unidas (ONU) cuyo objetivo fue el de recopilar casos éxitos de RSE, se sumaron a los del Banco Interamericano de Desarrollo (BID) que ha financiado proyectos para el desarrollo de normas nacionales y regionales de RSE, también organismos como la International Standarization Organization (ISO), desde el 2005 hasta el 2011, ha trabajado en la generación de la denominada ISO 26000, pero manteniéndose el concepto del Libro Verde donde la RSE seguía siendo de aplicación "voluntaria para las empresas" según "Comunicación puesta en marcha de una asociación para el crecimiento y la creación de empleo: hacer de Europa un polo de excelencia sobre Responsabilidad Social Corporativa, de 22 de marzo de 2006." (Bardel, 2019, p. 42) 
Pero es a partir del 2011 con el Comunicación "Puesta en marcha de una asociación para el crecimiento y la creación de empleo: hacer de Europa un polo de excelencia sobre Responsabilidad Social Corporativa", de 22 de marzo de 2006. (Bardel, 2019, p. 45) que la responsabilidad social pasa a ser de una ley suave (soft Law) a una ley rigurosa (Hard Law), y cambia el concepto de RSE de voluntario al siguiente se considera RSE "la responsabilidad de las empresas por su impacto en la sociedad" (Bardel, 2019, p. 45), adicionalmente se suma La Directiva 2014/95 del Parlamento Europeo y del Consejo, que introduce y regula el informe no financiero, convirtiéndose en obligatorio para las empresas, dentro de la Unión Europea.

Pero que sucede en América Latina, en Asia y en África, las organizaciones están interesadas en la responsabilidad social de las empresas en la medida en que ésta puede contribuir positivamente a sus objetivos estratégicos. Y todavía a nivel legal se sigue considerando a la responsabilidad social como un compromiso "voluntario de las empresas", para asegurar un crecimiento económico, social y ambientalmente responsable y por lo cual la generación de acciones sigue siendo considerados una carga que para las empresas (Villacís, Suarez, \& Güillín, 2016).

Adicionalmente, la Responsabilidad Social en América Latina y sobre todo entre los empresarios ecuatorianos se la suele confundir con filantropía. Es así que cuando se piensa en empresas socialmente responsables se trata de aquellas que son capaces de implementar una serie de prácticas relacionadas con aspectos tales como: buen gobierno dentro de la organización, desarrollo integral de sus colaboradores en el ámbito laboral e incluso en el familiar; desarrollo de proveedores, a quienes se los debe considerar como socios en su cadena de valor; buen relacionamiento con clientes y consumidores e incluso con la comunidad en la que opera la empresa; cuidado del ambiente; y aplicación de principios de derechos humanos, lo cual es transversal a las acciones de las empresas.

Según, Torresa (2012) menciona que más del 50\% de las empresas ecuatorianas se interesa sustancialmente por derechos humanos, prácticas laborales y responsabilidad del producto. Un menor número de empresas se preocupa por temas como proveedores o gobernanza de la organización.

Partiendo de ellos lo verdaderamente importante en las empresas es ser socialmente responsables, es así que los empresarios ecuatorianos conciben a la responsabilidad social como un modelo de gestión que pasa por varios procesos: planificación, ejecución, monitoreo y, finalmente, evaluación.

Actualmente se cuenta con distintos instrumentos para facilitar la implementación de la responsabilidad social y la medición de los resultados de ésta. Es así como se dispone de la Guía G3 del Global Reporting Initiative (GRI), como una metodología para elaborar un reporte de sostenibilidad.

Es de vital importancia para las empresas contar con un documento que contenga indicadores de medición de responsabilidad social, lo cual facilitaría el proceso de comunicación de los avances de la empresa en temas de responsabilidad social a los diferentes grupos de interés.

Lamentablemente en el país es bajo el porcentaje de empresas que disponen de un reporte o informe de sostenibilidad o de un documento similar. Es menor aún la cantidad de reportes elaborados con base en la metodología del GRI.

Considerando que menos del 1\% de las memorias de las empresas ecuatorianas serían elaboradas con la metodología del GRI, existe una gran preocupación sobre todo por parte de los empresarios imbabureños, quienes no disponen de un modelo de RSE eficiente y sustentable que les permita satisfacer los requerimientos tanto sociales como empresariales.

Responsabilidad social: factor clave de la gestión de los recursos humanos en las organizaciones del siglo XXI.

Según (Cornejo, 2009) una empresa es responsable cuando alcance los siguientes aspectos: Ofrecer productos y servicios que respondan a necesidades de sus usuarios, contribuyendo al bienestar, tener un comportamiento que vaya más allá del cumplimiento de los mínimos reglamentarios, optimizando en forma y contenido la aplicación de todo lo que le es exigible, aplicar la ética en todas las decisiones de directivos y personal con mando, y formar parte fundamental de la cultura de empresa, así mismo las relaciones con los trabajadores han de ser prioritarias, asegurando unas condiciones de trabajo seguras y saludables, respetar con dedicación el medio ambiente y atender a las necesidades de la comunidad en general.

Sin embargo, es lamentable que las empresas de Imbabura no logren cumplir con los requerimientos antes mencionados, ya que no disponen de una herramienta que les direccione hacia el cumplimiento de prácticas socialmente responsables y tampoco han aplicado indicadores para la evaluación y seguimiento de la gestión de RSE 


\section{METODOLOGÍA}

\section{Diseño de la metodología}

Para el presente estudio, según Hernández, et. al. (2014) el enfoque investigativo más adecuado para comprobar una hipótesis es el cuantitativo, y el tipo de alcance definido es el exploratorio, las técnicas utilizadas para la recolección de los datos se fundamenta en la medición del número de empresas que integran la responsabilidad social empresarial en su filosofía estratégica, y el número de gerentes y trabajadores que manifestaron a través de una encuesta su percepción acerca de la ejecución.

Los métodos que se utilizaron fueron el analítico sintético para resumir la bibliografía; el histórico lógico para fundamentar la trayectoria del antecedente de la responsabilidad social empresarial y el método inductivo y deductivo para analizar los resultados obtenidos a través de la encuesta.

\section{Procedimientos de muestreo}

\section{Criterio de selección de la población y muestra.}

La población seleccionada fueron todas las empresas que en la actualidad se encuentren activas, para lo cual se acudió a la base de datos de las Cámaras de Comercio de la Provincia, al del Servicio de Rentas Internas del Ecuador, a las Municipalidades de Antonio Ante, Cotacachi, Ibarra, Otavalo, Pimampiro, Urcuquí, en la Provincia de Imbabura-Ecuador, así como las del directorio de empresas del Instituto Nacional de Estadísticas y Censos del Ecuador, llegando a determinar que existen datos poblacionales hasta el año 2017, por lo que fue necesario proyectar la población para el año 2018, utilizando el método de lo mínimos cuadrados

\section{Tabla 1}

Proyección de las Empresas

\begin{tabular}{|c|c|c|c|c|}
\hline \multirow{2}{*}{ Año } & período & Número de Empresas & Variables & \\
\cline { 2 - 5 } & $\mathrm{x}$ & $\mathrm{y}$ & $\mathrm{X}^{2}$ & $\mathrm{XY}$ \\
\hline 2014 & 1 & 39867 & 1 & 39867 \\
2015 & 2 & 44887 & 4 & 89774 \\
2016 & 3 & 49985 & 9 & 149955 \\
2017 & 4 & 54856 & 16 & 219424 \\
\hline$\Sigma$ & 10 & 189595 & 30 & $\mathbf{4 9 9} 020$ \\
\hline 2018 & \multicolumn{3}{|r}{59915} \\
\hline
\end{tabular}

Fuente: Instituto Nacional de Estadísticas y Censos, 2017.

$$
\begin{aligned}
& y=a+b x \\
& \bar{x}=\frac{x}{N}=2,5 ; \bar{y}=\frac{y}{N}=47398,5 \\
& b=\frac{\sum x y-N \bar{x} \bar{y}}{\sum x^{2}-N \bar{x}^{2}}=5006,50 \\
& a=y-b x=34882,5 \\
& y=59915
\end{aligned}
$$


Por medio de este método de proyección se determinó que la población para el año 2018 serían 59915 empresas activas, dato que ha servido como insumo para determinar la muestra para poblaciones finitas, para lo cual se aplica la siguiente fórmula:

$$
n=\frac{P \cdot Q \cdot N}{(N-1)\left[\frac{E^{2}}{K^{2}}\right]+P \cdot Q}
$$

Dónde:

$\mathrm{n}=$ tamaño de la muestra

$\mathrm{N}=$ tamaño de la Población

$\mathrm{P}=$ probabilidad de que el evento ocurra $(0,5)$ o $50 \%$

$\mathrm{Q}=$ probabilidad de que el evento no ocurra $(0,5)$ o $50 \%$

$\mathrm{E}=0,05$ o $5 \%$

$\mathrm{K}=1,96$

$$
\begin{gathered}
n=\frac{59915 \times 0,5 \times 0,5}{\left[(59915-1)\left(\frac{0,05^{2}}{1,96^{2}}\right)\right]+(0,5 \times 0,5)} \\
n=381,73 \cong 382
\end{gathered}
$$

Una vez aplicado la fórmula para muestras finitas se ha procedido ha distribuir para los cinco cantones a nivel provincial, estableciendo que la mejor técnica a utilizar es la encuesta por el tamaño de la muestra que es de 382, así como por la distancia de las empresas entre sí, otro factor a considerar fue el tiempo debido que está dirigido al personal administrativo y que su disponibilidad para una entrevista era muy limitada y en algunos casos no había ninguna posibilidad.

\section{Procedimiento para la selección de participantes}

La información recogida durante el diagnóstico, se la clasificó en partiendo de una base de datos catastrales por unidades primarias (cantones), unidades secundarias (clasificador industrial internacional uniforme versión 4.0), y unidades finales (empresas), número que se selecciona de acuerdo con el tamaño proyectado de las unidades primarias.

Otro aspecto, que se consideró son los negocios que al momento de realizar la investigación se encontraban activos, por lo que se eliminó del estudio a las empresas que al momento de verificar teléfonos, direcciones que no coincidían con las bases de datos proporcionados por los catastros municipales.

\section{Mediciones variables}

Para la presente investigación se tomó como referencia los conceptos sobre Responsabilidad Social Empresarial plasmados en la norma ISO 26000 considerando las siguientes variables. 


\section{Tabla 2}

Matriz de Operacionalización de Variables

\begin{tabular}{|c|c|c|c|c|}
\hline Variable & Dimensión & Tipo & Escala & Pregunta \\
\hline \multirow{12}{*}{$\begin{array}{l}\text { Gobernanza } \\
\text { de la } \\
\text { organización }\end{array}$} & \multirow{2}{*}{$\begin{array}{l}\text { Dirección } \\
\text { estratégica }\end{array}$} & Cuantitativa & Nominal & $\begin{array}{l}\text { Desarrolla estrategias, objetivos y metas que reflejen } \\
\text { su compromiso hacia la responsabilidad social. }\end{array}$ \\
\hline & & Cuantitativa & Nominal & $\begin{array}{l}\text { Demuestra compromiso y rendición } \\
\text { de cuentas por parte de los líderes. }\end{array}$ \\
\hline & \multirow{2}{*}{$\begin{array}{l}\text { Crear una } \\
\text { atmósfera }\end{array}$} & Cuantitativa & Nominal & $\begin{array}{l}\text { Crea y promueve un ambiente en los que se practiquen } \\
\text { los principios de laresponsabilidad social. }\end{array}$ \\
\hline & & Cuantitativa & Nominal & $\begin{array}{l}\text { Crea un sistema de incentivos económicos y no } \\
\text { económicos asociados al desempeño en } \\
\text { responsabilidad social. }\end{array}$ \\
\hline & \multirow{3}{*}{$\begin{array}{l}\text { Nutrir la } \\
\text { atmósfera }\end{array}$} & Cuantitativa & Nominal & $\begin{array}{l}\text { Usa eficientemente los recursos financieros, } \\
\text { naturales y humanos. }\end{array}$ \\
\hline & & Cuantitativa & Nominal & $\begin{array}{l}\text { Promueve oportunidades justas para que los grupos } \\
\text { minoritarios, (incluyendo mujeres y grupos raciales } \\
\text { y étnicos), puedan ocupar cargos directivos de } \\
\text { la organización. }\end{array}$ \\
\hline & & Cuantitativa & Nominal & $\begin{array}{l}\text { Equilibra las necesidades de la organización y sus } \\
\text { partes interesadas, incluidas las necesidades } \\
\text { inmediatas y aquellas de las generaciones futuras. }\end{array}$ \\
\hline & \multirow{3}{*}{$\begin{array}{l}\text { Promover la } \\
\text { participación }\end{array}$} & Cuantitativa & Nominal & $\begin{array}{l}\text { Establece procesos de comunicación en dos } \\
\text { direcciones con sus partes interesadas, identificando } \\
\text { áreas de acuerdo y desacuerdo y negociando para } \\
\text { resolver posibles conflictos. }\end{array}$ \\
\hline & & Cuantitativa & Nominal & $\begin{array}{l}\text { Promueve la participación eficaz de los empleados de } \\
\text { todos los niveles, en las actividades de la organización } \\
\text { relacionadas con la responsabilidad social.los } \\
\text { empleados de todos los niveles, }\end{array}$ \\
\hline & & Cuantitativa & Nominal & $\begin{array}{l}\text { Equilibrar el nivel de autoridad, responsabilidad y } \\
\text { capacidad de las personas que toman decisiones } \\
\text { en representación de la organización. }\end{array}$ \\
\hline & \multirow{2}{*}{ Monitoreo } & Cuantitativa & Nominal & $\begin{array}{l}\text { Mantiene registro de la implementación de las } \\
\text { decisiones para asegurar que dichas decisiones se } \\
\text { llevan a cabo de manera socialmente responsable. }\end{array}$ \\
\hline & & Cuantitativa & Nominal & $\begin{array}{l}\text { Revisa y evalúa, periódicamente, los procesos de } \\
\text { gobernanza de la organización; adaptar los procesos } \\
\text { en función del resultado de las revisiones y comunicar } \\
\text { los cambios a toda la organización. }\end{array}$ \\
\hline
\end{tabular}

1 En la encuesta se cambió por "Prácticas administrativas" para que sea más entendible. 


\title{
Procedimiento para recoger la información
}

El procedimiento muestral, que mejor se adaptó fue el probabilístico polietápico, con la selección al azar de las unidades primarias (cantones), de las unidades secundarias (parroquias) y las unidades finales (personas).

En la primera etapa se dividió la muestra calculada por actividad principal de acuerdo con la Clasificación Industrial Internacional Uniforme de todas las actividades económicas (CIIU). Posteriormente se distribuyó por cantones, parroquias y barrios en forma proporcional al catastro de cada cantón.

En la segunda etapa se seleccionó una muestra simple de los negocios identificados en las parroquias de cada uno de los seis cantones que conforman la provincia de Imbabura.

En una siguiente etapa de acuerdo con los sectores seleccionados, se aplicó al número de empresas que se estableció en la tabla de distribución de la muestra a través un procedimiento aleatorio contabilizando cada enésima vez, si el sector lo ameritaba. En caso de rechazo, inexistencia o inexactitud de las direcciones de las empresas, u otros impedimentos para realizar la encuesta, la empresa seleccionada fue remplazada, siguiendo el procedimiento aleatorio de cada enésima vez.

Finalmente, la cuarta etapa consistió en aplicar la encuesta a las unidades finales. Las empresas encuestadas fueron seleccionadas por su cercanía a los sectores empresariales de forma aleatoria tanto en el sector urbano como en el sector rural, a través de una tabla de asignación adecuada para cada cantón. Al encuestado elegido se aplicó un filtro para conocer si habita en el sector, de no ser así se finalizaba la encuesta.

\section{RESULTADOS}

\author{
Análisis inferencial del estudio. Planteamiento de la hipótesis nula de la percepción de las prácti- \\ cas administrativas socialmente responsables.
}

La tratar de contestar la siguiente pregunta ¿La percepción de prácticas administrativas socialmente responsables depende de la rendición de cuentas por parte de los líderes?, La hipótesis parte del siguiente supuesto donde Ho: La rendición de cuentas es independiente de la percepción de las prácticas administrativas socialmente responsables de los gerentes, caso contrario H1: La rendición de cuentas depende de la percepción de las prácticas administrativas socialmente responsables de los gerentes, para lo cual es necesario determinar su dependencia, y por lo tanto no están relacionas entre sí. Considerando que para esta investigación se planteó un nivel de confianza del 95\% lo que equivale a un margen de error del $5 \%$. La comprobación de las hipótesis se aplicó a través de la prueba del Chi-Cuadrado, para lo cual se planteó la siguiente relación para la hipótesis nula Ho: p-valor < 0,05, y para la hipótesis alterna $\mathrm{H} 1$ : p-valor > 0,05.

Variable independiente: rendición de cuentas.

Ho: p-valor $<0,05$
H1: p-valor $>0,05$

\section{Prueba de significación de la hipótesis}

Con el propósito de contestar los supuestos planteados en este estudio, se ha recolectado datos de una muestra de 382 observaciones, información que se usará para comprobar que tan probable es el parámetro de población hipotético con la afirmación del supuesto. 
La prueba que se ha utilizado para comprobar la asociatividad entre las variables es la chi-cuadrado, es decir probar si más de dos proporciones de la población pueden ser consideradas iguales.

¿La rendición de cuentas depende de la percepción de práctica administrativas socialmente responsables?

El resultado demuestra que la hipótesis nula debe rechazarse, debido a que el chi-calculado es mayor que el chi-critico, de acuerdo con los resultados de la prueba del chi-cuadrado obtenido en SPSS versión 23, este indica que la chi-calculada es de 175,82 en contraste al resultado de la chi-crítica que es de 3,84. Éste último resultado se obtuvo buscando en la tabla de la chi-cuadrada con un margen de error del 5\% y 1 grado de libertad.

\section{Tabla 3}

Tabla Cruzada Demuestra Compromiso y Rendición de Cuentas por parte de los Gerentes.

*Implementación de prácticas administrativas socialmente responsable

\begin{tabular}{|c|c|c|c|c|c|}
\hline \multicolumn{3}{|l|}{ Variables } & \multicolumn{3}{|c|}{$\begin{array}{l}\text { Implementación de prácticas } \\
\text { administrativas socialmente responsable }\end{array}$} \\
\hline & & & NO & SI & Total \\
\hline \multirow{4}{*}{$\begin{array}{l}\text { Demuestra compromiso y } \\
\text { rendición de cuentas por } \\
\text { parte de los gerentes. }\end{array}$} & \multirow{2}{*}{ NO } & Recuento & 234,00 & 49,00 & 283,00 \\
\hline & & Recuento esperado & 179,30 & 103,70 & 283,00 \\
\hline & \multirow{2}{*}{ SI } & Recuento & 8,00 & 91,00 & 99,00 \\
\hline & & Recuento esperado & 62,70 & 36,30 & 99,00 \\
\hline \multirow{2}{*}{\multicolumn{2}{|c|}{ Total }} & Recuento & 242,00 & 140,00 & 382,00 \\
\hline & & Recuento esperado & 242,00 & 140,00 & 382,00 \\
\hline
\end{tabular}

Fuente: elaboración propia. 
Tabla 4

Pruebas de Chi-cuadrado

\begin{tabular}{|l|c|c|c|c|c|}
\hline & Valor & gl & $\begin{array}{c}\text { Significación } \\
\text { asintótica } \\
\text { (bilateral) }\end{array}$ & $\begin{array}{c}\text { Significación } \\
\text { exacta } \\
\text { (bilateral) }\end{array}$ & $\begin{array}{c}\text { Significación } \\
\text { exacta } \\
\text { (unilateral) }\end{array}$ \\
\hline $\begin{array}{l}\text { Chi-cuadrado de Pearson } \\
\text { Corrección de continuidad }\end{array}$ & $175,82^{\mathrm{a}}$ & 1 &, 00 &, 00 &, 00 \\
$\begin{array}{l}\text { Razón de verosimilitud } \\
\text { Prueba exacta de Fisher }\end{array}$ & 185,62 & 1 & 1 &, 00 &, 00 \\
$\begin{array}{l}\text { Asociación lineal por lineal } \\
\text { N de casos válidos }\end{array}$ & 175,36 & 1 &, 00 & &, 00 \\
\hline
\end{tabular}

a. 0 casillas $(0,0 \%)$ han esperado un recuento menor que 5 . El recuento mínimo esperado es 36,28.

b. Sólo se ha calculado para una tabla $2 \times 2$

Fuente: elaboración propia.

Por otra parte, si tomamos el criterio del p-valor, el planteamiento indica que debe rechazarse la Hipótesis nula, si el p-valor (Significación asintótica) que se obtiene en el SPSS versión 23, en este caso 0.00 es menor que el margen de error que corresponde al 5\%, (0.05) este debe rechazarse; por lo tanto, la hipótesis nula planteada se rechaza, y se puede decir que la ejecución de consumo están relacionadas con la planificación de las prácticas administrativas. 


\section{DISCUSIÓN DE LOS RESULTADOS}

El análisis del estudio se basa en los datos obtenidos de la recolección de datos de una muestra poblacional, con el propósito de comprobar si los parámetros hipotéticos recogidos a través de un grupo de preguntas dicotómicas son independientes entre sí, para lo cual se plantean las siguientes hipótesis:

Ho: La rendición de cuentas es independiente de la percepción de las prácticas administrativas socialmente responsables de los gerentes.

H1: La rendición de cuentas depende de la percepción de las prácticas administrativas socialmente responsables de los gerentes.

Esta hipótesis nula debe rechazarse si el chi-calculado es mayor que el chi-critico, de acuerdo a los resultados de la prueba del chi-cuadrado obtenido en SPSS, este indica que la chi-calculada es de 175,82 en contraste al resultado de la chi-crítica que es de 3,841 . Éste último resultado se obtuvo buscando en la tabla de la chi-cuadrada con un margen de error del $5 \%$ y 1 grado de libertad.

Por otra parte, si tomamos el criterio del p-valor, el planteamiento indica que debe rechazarse la Hipótesis nula, si el p-valor (Significación asintótica) que se obtiene en el SPSS es menor que el margen de error que corresponde al $5 \%$. A continuación, se procedió a calcular el p-valor en el SPSS dando como resultado 0,000 < 0,05 por lo tanto la hipótesis planteada se rechaza, y se puede decir que las prácticas administrativas socialmente responsables están relacionadas con la filosofía estratégica.

Existen varios trabajos que han investigado acerca de la responsabilidad social empresarial y han profundizado en la materia de gobernanza organizacional, como uno de esos trabajos relevantes se encuentra el Mancilla y Saavedra (2015), y explica la relación existente entre la responsabilidad social y las reglas de control interno que las empresas establecen en sus organizaciones y como su principal hallazgo es que las empresas estudiadas muestran un cumplimiento de la normatividad por obligación.

Otro trabajo relevante es el de Martínez, et al (2015) que es un estudio sobre el consejo de administración y sus prácticas en responsabilidad social empresarial, la materia en la que profundiza es la de gobernanza organizacional, como uno de sus conclusiones es que el comportamiento socialmente responsable está vinculado a la independencia y diversidad del consejo.

Por otra parte, Giraldo (2008) presenta un informe acerca de sus resultados de su investigación referente a si existe una relación entre la estrategia organizacional y la responsabilidad social empresarial, y se concluye que hay un desconocimiento acerca del tema y que no incluyen a la responsabilidad social empresarial como parte de la estrategia.

Luego de recoger los datos para el presente estudio empírico, y de comprobar que existe una relación entre las variables a través de la prueba del chi-cuadrado; se procedió a realizar el respectivo análisis de la estadística descriptiva de las variables correlacionadas, en donde se determinó que las empresas no aplican prácticas administrativas con responsabilidad social; adicionalmente a esta afirmación se suma la evidencia recogida durante la evaluación de las variables que han sido definidas en la ISO 26000 tales como: (1) filosofía estratégica, (2) rendición de cuentas, (3) 
ambiente organizacional, (4) procesos de comunicación, y (5) participación de los empleados, demostrando que este tipo de prácticas no son de uso general en el sector empresarial de Imbabura.

La explicación de porque no se aplica este tipo de prácticas basados en la responsabilidad social se la puede tratar desde el punto de vista de la Teoría de los Costos de Transacción, Arrow (1974 citando por Brunet, 2011) realiza una pregunta ¿.para qué coordinar actividades si los costos de recurrir al mercado son nulos? Es decir que rentabilidad obtiene un empresario por iniciar actividades de responsabilidad social si el costo que conlleva puede ser alto y la rentabilidad nula.

Así mismo se puede explicar este problema a través de la teoría de recursos y capacidades, en donde "Los recursos son medios aislados, necesarios pero no suficientes para alcanzar los fines de la empresa, mientras que las capacidades integran recursos de diferente naturaleza para llevar a cabo actividades productivas" (Brunet, 2011, p. 152), lo que quiere decir es que las empresas deben disponer de recursos y de capacidades para aplicar correctamente las prácticas administrativas con responsabilidad social, y que de acuerdo a la evidencia recogida la mayor parte de las empresas del sector Imbabureño no las poseen.

Así mismo la teoría de la contingencia es otro factor determinante para que las empresas de Imbabura apliquen actividades de responsabilidad social, ya que estos depende de factores contingentes, es decir que la empresa responde a factores externos propios del dinamismo económico de la cultura y del sector a la que pertenece, basados en oportunidades y amenazas que se presenten a los empresarios, quienes prefieren elaborar una estrategia en respuesta a estos factores externos, y no formular estrategias como parte de la conciencia ética en favor del entorno natural y social que le rodea.

Finalmente, la teoría de la apropiabilidad influye en la actitud de los empresarios, de la Cruz (2007) expone que "las empresas pueden disminuir o anular totalmente las inversiones en desarrollo social, porque la apropiabilidad en inversiones sociales puede ser muy escasa y no recibirían los beneficios deseados de la inversión", teoría que va de la mano con la de los costos de transacción. Sobre todo, tomando en cuenta la te

ndencia de la conducta racional del inversor que Markowitz (1957) planteo en su hipótesis “, el inversor prefiere aquellas carteras con una mayor rentabilidad y un menor riesgo”, por lo tanto, un empresario imbabureño, invertirá en proyectos que sean de fácil retorno, y mayor movimiento transaccional, que por lo general serán proyectos de compra y no desarrollo social.

\section{REFERENCIAS}

Bardel, D. (2019). Una aproximación al desarrollo de la responsabilidad social empresarial en la Unión Europea: Rendición de cuentas y normas de derecho internacional privado. Revista de Estudios Europeos(73), 34-65.

Brunet, I. (2011). Creación de Empresas: emprendimiento e innovación . Bogotá: Ediciones de la U.

Carroll, A. (1979). A Three-Dimensional Conceptual Model of Corporate Performance. Academy of management(4), 497505. doi:https://doi.org/10.5465/amr.1979.4498296

Cochran, P., Wood, R., \& Jones, T. (1985). The Composition of Boards of Directors and Incidence of Golden Parachutes. Academy of Management, 28(3), 664-671. doi:https://doi.org/10.5465/256121 
Cornejo, A. (2009). Responsabilidad Social: Factor Clave de la Gestión de los Recursos Humanos en las organizaciones del siglo XXI. Revista Crítica de Ciencias Sociales y Jurídicas. Vol. 2, n.o 1, pp. 2-17.

Correa, J. G. (2007). Evolución histórica de los conceptos de responsabilidad social empresarial y balance social. Semestre Económico, 10(20), 87-102.

Cruz, C. (2007). Los determinantes y las consecuencias de las estrategias de innovación; un análisis del sector manufacturero y de servicios de España (Tesis doctoral). Universidad Autónoma de Barcelona. Ballaterra.

Donaldson, T., \& Preston, L. (1995). The stakeholder theory of the corporation: concepts, evidence, and implications. Academy of Management Review, 20(1), 65-91.

Drucker, P. (1984). Converting Social Problems into Business Opportunities: The New Meaning of Corporate Social Responsibility. California Management Review, 26(2), 53. Obtenido de https://search.proquest.com/openview/8dde78b$449 \mathrm{~d} 8452 \mathrm{c} 447619 \mathrm{ddb} 82 \mathrm{~d} 15 \mathrm{f9} / 1$ ? pq-origsite $=$ gscholar\&cbl $=42246$

Friedman, M. (1970). The social responsability of business is toincrease its profits. New York Times Magazine, 122-126.

Giraldo, G. (2008). Responsabilidad social empresarial en Antioquia. Revsita Universidad EAFIT, 44(1), 38-59.

Hernández, R., Fernández, C., \& Baptista, P. (2014). Metodología de la investigación (6a. ed.). México: McGraw- Hill.

Kotler, P., \& Lee, N. (2005). Best of Breed: When it Comes to Gaining a Market Edge While Supporting a Social Cause, "Corporate Social Marketing" Leads the Pack. Social Marketing Quarterly, 11(3-4), 91-103. doi:https://doi. org/10.1080/15245000500414480

Kramer, M., \& Porter, M. (2002). La ventaja competitiva de la filantropía corporativa. Harvard Bussiness Review, 80(12), 4962. Obtenido de https://dialnet.unirioja.es/servlet/articulo?codigo $=1431332$

Mancilla, M., \& Saavedra, M. (2015). El gobierno corporativo y el comité de auditoría en el marco de la responsabilidad social empresarial. Contaduría y Administración, 60(1), 486-506.

Martínez, J., Vaquero, L., Cuadrado, B., \& García, I. (2015). El gobierno corporativo y la responsabilidad social empresarial corporativa en el sector bancario: el papel del consejo de administración. Investigaciones Europeas, de Dirección y Economía de la Empresa, 21(1), 129-138.

Markowitz, H.M. and Manne, A.S. (1957) On the Solution of Discrete Programming Problems. Econometrica, 25, 84-1 10. Published By: The Econometric Society.

Monroy, G. (2013). Estado del Arte de la RSE y su aplicabilidad al sector energía renovable y electricidad de Guatemala, Honduras y Nicaragua. Guatemala: OLADE.

Raufflet, E., Lozano, J., Barrera, E., \& García, C. (2012). Responsabilidad Social Empresarial. México: Pearson Educación.

Sethi, S. P. (1975). Dimensions of Corporate Social Performance: An Analytical Framework. California Management Review, 17(3), 58-64. doi:https://doi.org/10.2307/41162149

Torresa, M. (2012). Estudio de Responsabilidad Social de Empresas en Ecuador.

Varela, L. V. (2013). Estado del arte de la responsabilidad social. Gestión y desarrollo, 10(1), 55-73.

Villacís, C., Suarez, Y., \& Güillín, X. (2016). Análisis de la Responsabilidad Social en el Ecuador. Revista Publicando, 3(8), 452-466.

Wood, D. (1991). Corporate Social Performance Revisited. Academy of Management(16), 691-718. doi:https://doi. org/10.5465/amr.1991.4279616 